\title{
Spatial Beam Shaping for Femtosecond Laser in Vitreo-Retinal Sur- gery
}

\author{
A. Hansen ${ }^{1}$, T. Ripken ${ }^{1}$, A. Krüger ${ }^{1}$ \\ ${ }^{1}$ Laser Zentrum Hannover (Biomedical Optics Department), Hanover, Germany, A.Hansen@lzh.de
}

\begin{abstract}
In ophthalmic surgery, femtosecond laser pulses provide a high precision tool for tissue dissection in the anterior part of the eye with little peripheral implications. However, for an application in the posterior part the precision is diminished by the aberrations of the eye which also cause higher pulse energy required for photodisruption. In order to increase the precision and decrease the pulse energy for vitreo-retinal surgery an adaptive optics system was designed for spatial beam shaping of femtosecond laser pulses. A Hartmann-Shack-Sensor was used for aberration measurement and a deformable mirror for aberration correction. The influence of an adaptive optics aberration correction on the resulting wave front and incision process was investigated in an eye model. The adaptive optics system designed for the requirements of vitreoretinal surgery provides a wave front correction with diffraction limited precision. The spatial confinement as a consequence of the corrected wave front decreases the pulse energy for the incision process in the model eye. As less energy is required for the incision process adaptive optics in a femtosecond laser scalpel potentially allow for tissue dissection with reduced peripheral damage. This offers new possibilities for non-invasive high precision surgery in the posterior eye segment and the treatment of vitreal and retinal pathologies.
\end{abstract}

\section{Introduction}

In ophthalmic surgery, femtosecond laser have become an established tool for high precision tissue dissection in numerous applications in the anterior part of the eye [1]. Currently, the two major applications in the cornea are the femto-LASIK (laser in situ keratomileusis) where the femtosecond laser has replaced the microkeratome for corneal flap creation and corneal transplantations (penetrating or lamellar keratoplasty) while other applications include corneal implants or corneal biopsies. More recent developments have made the lens accessible as a target for femtosecond laser surgery. Applications in the lens include fragmentation of the lens for cataract treatment, capsulotomy, or enhancement of lens flexibility in presbyopia treatment. Due to the success of femtosecond laser applications in the anterior part of the eye a logical consequence is to target structures in the posterior part of the eye for femtosecond laser surgery. Possible applications include retinal, vitreo-retinal and vitreal pathologies. The current standard surgical procedure for the posterior segment, the vitrectomy, is an invasive process accompanied by vitreous humor removal and inevitably followed by cataract formation due to the mechanical trauma on the lens. A noninvasive laser procedure could potentially eliminate the need for vitrectomy and thus prevent complications like cataract formation or inflammation. The optical scalpel for ophthalmic surgery with femtosecond laser pulses uses the laser induced optical breakdown (LIOB) for tissue disruption [2]. When a tissue specific threshold irradiance is surpassed nonlinear absorption processes lead to a free electron density high enough to ignite a plasma which then causes a cavitation bubble to form, rise, and collapse. As a consequence of the cavitation bubble process the tissue is locally disrupted. Since femtosecond laser pulses provide the pulse energy over a shorter period of time than pico- or nanosecond laser pulses the threshold irradiance for LIOB can be surpassed for lower pulse energies. Additionally, as the time for thermal energy transfer from the plasma to the tissue is longer than the pulse duration the thermal energy deposition in the tissue is minimal. This implies that peripheral implications are reduced which is especially important in the vicinity of the retina. However, the irradiance is not only dependent of the irradiation time and pulse energy but also of the irradiated area. A perfect eye focusses the incident light to a diffraction limited spot on the retina, but in a typical eye aberrations cause a distortion of the focus. This reduces the precision and also raises the required pulse energy therefore increasing the risk for peripheral implications. An adaptive optics system can provide aberration correction by measuring the specific aberrations of the eye with a wave front sensor and correcting for them with a wave front modulator. With the aberrations corrected the incident light is again focused to a diffraction limited spot as has been shown for imaging devices like fundus cameras [3], scanning laser ophthalmoscopes [4], and optical coherence tomography [5]. With diffraction limited spot size the required pulse energy for LIOB is minimized offering new possibilities for microsurgery in the posterior part of the eye. In this work we present an adaptive optics system with an integrated femtosecond laser pulse source that enables for the correction of aberrations in a model eye. This system is intended to spatially confine the laser pulse energy and therefore decrease the pulse energy for the incision process in vitreo retinal surgery. 


\section{Methods}

The design of the adaptive optics system with integrated optical femtosecond laser pulse scalpel is schematically depicted in image 1 .

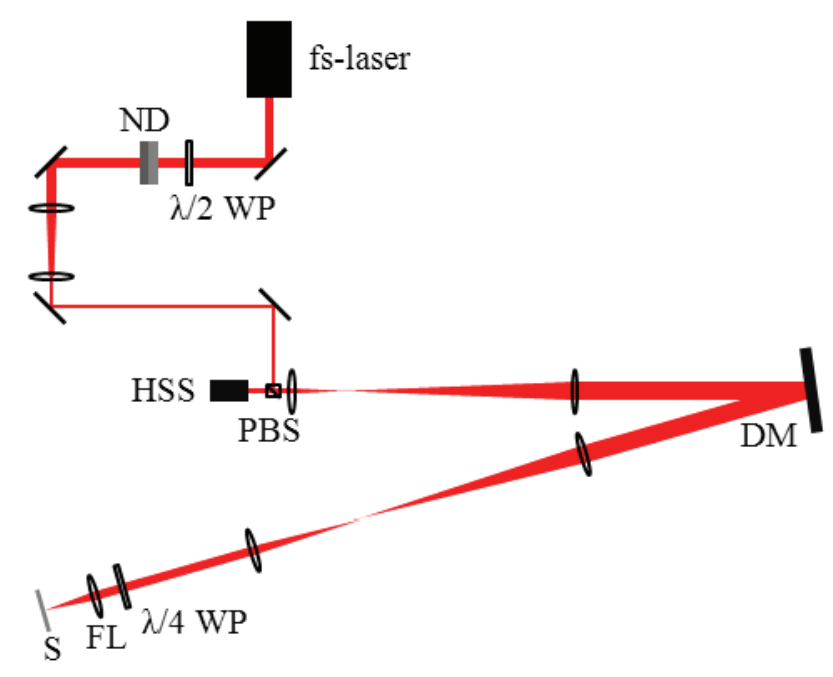

Image 1 Adaptive optics system design with integrated femtosecond laser pulse source (DM-deformable mirror, FL-focusing lens, HSS-Hartmann-Shack-sensor, NDneutral density filter, PBS-polarizing beam splitter, Ssample, WP-wave plate)

The adaptive optics system comprises a wave front sensor, a wave front modulator, and a femtosecond laser pulse source as main components. The wave front sensor is a Hartmann-Shack-sensor (HASO32, Imagine Eyes, Orsay, France) with a microlens array of $40 \times 32$ lenslets over an aperture of $4.5 \mathrm{~mm} \times 3.6 \mathrm{~mm}$. The aberration measurement is performed with the same femtosecond laser source which is used for the LIOB so the measurement and the processing light share the same light pass. This precludes non common path aberrations due to non-coaxial alignment or due to different chromatic aberrations. The wave front modulator is an electromagnetic deformable mirror with a continuous circular flexible mirror membrane which is deformed by 52 actuators over a diameter of $15 \mathrm{~mm}$ (mirao 52-e, Imagine Eyes, Orsay, France). In order to minimize intensity fluctuations when applying a wave front modulation the deformable mirror needs to be optically conjugated to the Hartmann-Shack-sensor. Furthermore, imaging the sensor onto the mirror requires matching the apertures of both elements which is obtained with a Keplerian telescope. The deformable mirror is positioned at the minimum possible angle in order to maximize the useable diameter of the aperture. Another Keplerian telescope images the deformable mirror into the iris of the eye. As a femtosecond laser is used as light source all lenses are achromatic lenses in order to minimize chromatic aberrations in the system. The femtosecond laser source is a Spitfire Pro (Spectra Physics, Mountain View, CA, USA) TiSa regenerative amplifier. The interface between the laser and the adaptive optic system is realized by a polarizing beam splitter cube which in combination with a half wave plate also served as attenuation. Larger attenuation steps where achieved using neutral density filters. Selecting a specific polarization is advantageous when considering the reflection management of the design. Although the lenses are provided with an anti-reflection coating for the laser wavelength the remaining reflections still disturb the measurement signal as the signal is up to two orders of magnitude less intense than the reflections. In combination with a quarter wave plate in front of the eye the polarizing beam splitter enables separating the reflections from the measurement signal. The only remaining reflections are from the eye itself as it is positioned behind the wave plate. These reflections can be eliminated by either tilting the eye - here the focusing lens of the model eye - slightly or by placing an iris in the focal point of the first telescope. The adaptive optics system is operated in a double pass configuration. The light from the femtosecond laser pulse source is directed via the deformable mirror into the eye model. The backscattered light returns through the system to the Hartmann-Shack-sensor. The eye model consists of a plano-convex focusing lens which models the refractive power of both the cornea and the lens in a human eye. The retina is modeled by a sample with scattering surface, e.g. paper. For introducing aberrations to the eye model two different aberration samples were used. To model low order aberrations like astigmatism and defocus a cylindrical lens with long focal length compared to the focal length of the model eye was positioned in between the focusing lens and the sample. Although low order aberrations (tip, tilt, defocus, and astigmatism) are the more dominant aberrations typically found in human eyes, higher order aberrations - especially spherical aberration - are also significant. These higher order aberrations were modeled by using an object slide with a thin film of hairspray. For aberration correction, first, an interaction matrix was acquired to learn how the wave front is distorted when the deformable mirror surface is modified. The interaction matrix was then used to calculate a command matrix for setting specific target wave fronts in open loop or closed loop control. The software used was the Casao program (Imagine Eyes, Orsay, France). The resulting wave front as calculated from the slopes of the Hartmann-Shack-sensor signal was compared for the case of measuring the aberrations of the model eye and for the case of correcting for the measured aberrations in closed loop control. The criterion for wave front quality is the root mean square (rms) of the wave front over a pupil diameter of $7.5 \mathrm{~mm}$. Additionally the radiant exposure was acquired at the Hartmann-Shacksensor as an improved spatial beam shaping will result in a more localized scattering area at the retina of the model eye and therefore a raised amount of light collected by the system for the second pass. 


\section{$3 \quad$ Results}

The adaptive optics system designed for the requirements of vitreo-retinal surgery provides a wave front correction with diffraction limited precision. The aberration correction is demonstrated for the model eye consisting of a plano-convex focusing lens, paper, and for aberrations a cylincrical lens, and an object slide with a thin film of hairspray. The wave front of the femtosecond pulse laser after double pass through the system and the model eye is shown in image 2 in a false color representation. As the cylindrical lens has a relatively long focal length the dominant aberration next to the expected astigmatism is a strong defocus. The total rms of the wave front amounts to $1.768 \mu \mathrm{m}$. The higher order aberrations show an rms of $0.218 \mu \mathrm{m}$.
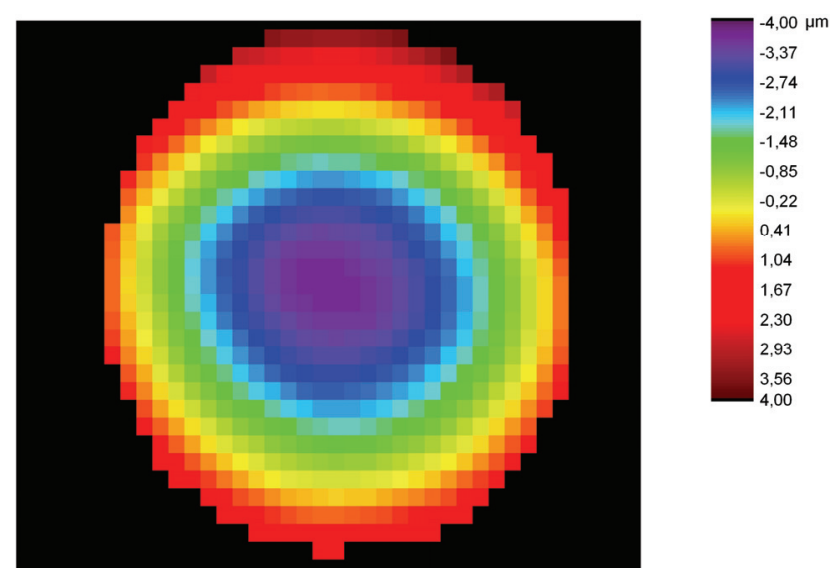

Image 2 Wave front aberration of model eye with low and high order aberrations

To correct for these aberrations the adaptive optics system is run in closed loop control. The resulting wave front of the femtosecond pulse laser after the correction is shown in image 3 in a false color representation. The aberrations from the model eye have been corrected for including both the strong low-order aberrations from the cylindrical lens as well as the high-order aberrations from the object slide
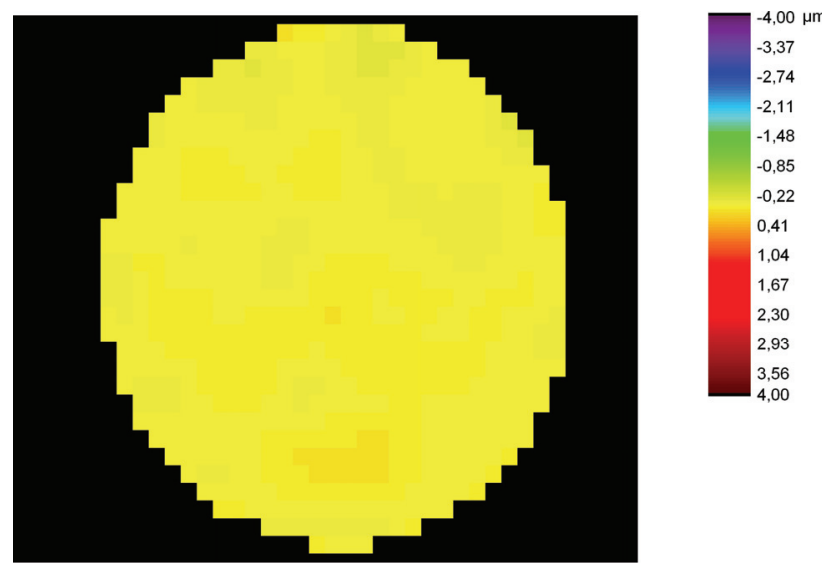

Image 3 Diffraction limited wave front of model eye after correction in closed loop with a thin film of hairspray. The resulting wave front shows a residual rms of $0.043 \mu \mathrm{m}$. The adaptive optics system therefore provides a wave front correction with diffraction limited precision. Another observation regards the radiant exposure on the Hartmann-Shack-sensor. When comparing the aberrated case with the corrected case the radiant exposure can be raised by a factor of 3 from the deformed wave front to the flat wave front. The spatial confinement as a consequence of the corrected wave front raises the irradiance at constant pulse energy in the model eye.

\section{Conclusion}

An adaptive optics system was designed for the requirements of vitreo-retinal surgery providing both the aberration correction in a double pass configuration and an interface for a femtosecond laser pulse source as an optical scalpel using the model eye's optics for focusing. The influence of the system's aberration correction in closed loop control was investigated in an eye model with both lower and higher order aberrations integrated in the model. It was shown that the system provides a wave front measurement and correction with diffraction limited precision for the presented aberrations. As a consequence of the corrected wave front the irradiance was raised at constant pulse energy. The raised irradiance is a result of the aberration correction in the first pass causing a more localized scattering area at the retina of the model eye and therefore a less diffuse collection of light in the second pass where the wave front is further corrected. Conversely, as we have shown before [6], the corrected wave front and the concurrent spatial confinement decrease the pulse energy required to surpass the threshold irradiance for the laser induced optical breakdown in the model eye. As less energy is required for the incision process adaptive optics in a femtosecond laser pulse scalpel potentially allow for tissue dissection with reduced collateral damage. Apart from the improved incision process an imaging device could also benefit from the diffraction limited aberration correction. An integrated imaging process would allow for precise tissue targeting and evaluation of outcome. The presented adaptive optics system for spatial beam shaping offers new possibilities for non-invasive high precision surgery in the posterior eye segment and the treatment of vitreal and retinal pathologies.

\section{Acknowledgment}

We acknowledge financial support for the project IKARUS (innovative cataract, age related presbyopia and retina treatment with ultrashort pulsed lasers; No. 13N11847) by the German Federal Ministry of Education and Research (BMBF). 


\section{References}

[1] Soong, H. K.; Malta, J. B.: Femtosecond Lasers in Ophthalmology. American Journal of Ophthalmology 147(2), 2009, pp. 189-197

[2] Vogel, A., Noack, J., Hüttman, G., and Paltauf, G.: Mechanisms of femtosecond laser nanosurgery of cells and tissues. Applied Physics B: Lasers and Optics 81, 2005, pp. 1015-1047

[3] Liang, J.; Williams, D. R.: Supernormal vision and high-resolution retinal imaging through adaptive optics. Journal of the Optical Society of America A 14(11), 1997, pp. 2884-2892

[4] Roorda, A.; Romero-Borja, F.; Donnelly III, W. J.; Queener, H.; Hebert, T. J.; Campbell, M. C. W.: Adaptive optics scanning laser ophthalmoscope. Optics Express 10(9), 2002, pp. 405-412

[5] Hermann, B.; Fernández, E. J.; Unterhuber, A.; Sattmann, H.; Fercher, A. F.; Drexler, W.; Prieto, P. M.; Artal, P.: Adaptive-optics ultrahigh-resolution optical coherence tomography. Optics Letters 29(18), 2004, pp. 2142-2144

[6] Hansen, A.; Ripken, T.; Heisterkamp, A.: Spatial Beam Shaping for Lowering the Threshold Energy for Femtosecond Laser Pulse Photodisruption. Proc. SPIE 8130-37, 2011 